\title{
The properties of early-type stars in the Magellanic Clouds
}

\author{
Christopher J. Evans \\ UK Astronomy Technology Centre, Royal Observatory Edinburgh, Blackford Hill, Edinburgh, \\ EH9 3HJ, UK \\ email: cje@roe.ac.uk
}

\begin{abstract}
The past decade has witnessed impressive progress in our understanding of the physical properties of massive stars in the Magellanic Clouds, and how they compare to their cousins in the Galaxy. I summarise new results in this field, including evidence for reduced mass-loss rates and faster stellar rotational velocities in the Clouds, and their present-day compositions. I also discuss the stellar temperature scale, emphasizing its dependence on metallicity across the entire upper-part of the Hertzsprung-Russell diagram.
\end{abstract}

Keywords. stars: early-type, stars: fundamental parameters, Magellanic Clouds

\section{Introduction}

The prime motivation for studies of early-type stars in the Magellanic Clouds over the past decade has been to quantify the effect of metallicity $(Z)$ on their evolution. The intense out-flowing winds in massive stars are thought to be driven by momentum transferred from the radiation field to metallic ions (principally iron) in their atmospheres; a logical consequence of this mechanism is that the wind intensities should vary with $Z$ (Kudritzki et al. 1987). Monte Carlo simulations predict that, for stars with $\mathrm{T}_{\text {eff }}>$ $25,000 \mathrm{~K}$, the wind mass-loss rates should scale with metallicity as $Z^{0.69}$ (Vink et al. 2000, 2001). This has a dramatic impact on their subsequent evolution. For example, an O-type star in the SMC should lose significantly less mass over its lifetime than a star in the Galaxy, thus retaining greater angular momentum. This could then lead to different late-phases of evolution such as the type of core-collapse supernova (SN), and offers a potential channel for long duration, gamma-ray bursts at low $Z$.

The $Z$-dependence of the initial rotational velocity distributions of massive stars and the importance of rotationally-induced mixing have also been active areas of research. For instance, Maeder et al. (1999) noted that the relative fraction of Be- to B-type stars increases with decreasing metallicity $\dagger$, suggesting that this might arise from faster rotational velocities at lower $Z$. The recent generation of evolutionary models has explored the effects of rotational mixing (e.g., Heger \& Langer 2000; Meynet \& Maeder 2000), with the prediction of larger relative surface-nitrogen enhancements at faster rotation rates, and at lower Z (Maeder \& Meynet 2001).

To date, we have lacked sufficient observations to explore the effects of metallicity thoroughly. Robust empirical results were needed with which to confront both the stellar wind and evolutionary models for early-type stars - here I summarise recent observations and quantitative analyses toward this objective.

$\dagger$ The sample of Maeder et al. comprised only one SMC cluster, NGC 330, long known to have a significant Be-fraction (e.g., Grebel et al. 1992) and sometimes suggested as a "pathological" case. However, new results from Martayan et al. (these proceedings) also find similarly large fractions for other SMC clusters. 


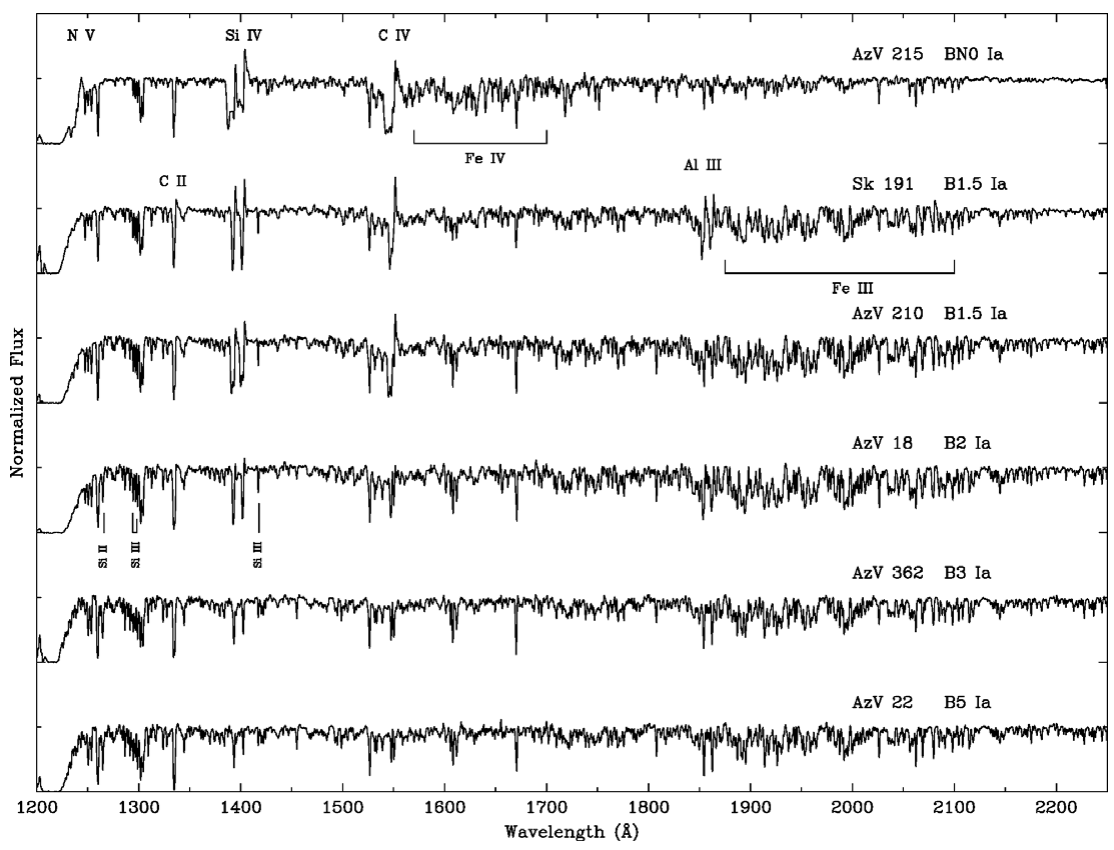

Figure 1. STIS spectra of B-type supergiants in the SMC (Evans et al., 2004a). Note the distinctive (although weak) P Cygni emission in the Nv, Si IV and C IV lines in AzV 215, and the iron "forests" which neatly illustrate the change in the predominant ionization stage in the early B-type domain.

\section{Unique insights from ultraviolet observations}

Ultraviolet (UV) spectroscopy provides an invaluable complement to optical spectroscopy in the determination of the physical parameters of early-type stars. The terminal velocity $\left(v_{\infty}\right)$ of the stellar wind can be measured from the saturated cores of the resonance lines, with information on the stratification and ionization of the wind provided by comparisons with model atmospheres. Unfortunately, only a handful of the brightest OB-type stars in the Clouds were within reach of high-dispersion observations with the International Ultraviolet Explorer (IUE).

The Hubble Space Telescope (HST) and the Far Ultraviolet Spectroscopic Explorer (FUSE) have provided essential observations of the winds of massive stars in the Clouds. Although limited by relatively low spectral resolution, the HST Faint Object Spectrograph (FOS) was used in the early 1990s to observe tens of stars in the Clouds. These spectra provided morphological evidence of weaker stellar winds in the SMC when compared to Galactic standards (Walborn et al. 1995), as well as estimates of $v_{\infty}$ (e.g., Prinja \& Crowther 1998). More recently, the HST Space Telescope Imaging Spectrograph (STIS) and FUSE have both delivered spectra at resolutions of $>10^{4}$, sufficient to resolve interstellar features clearly and to enable detailed comparisons with synthetic spectra.

STIS spectroscopy of 29 OB-type SMC stars was presented by Walborn et al. (2000) and Evans et al. (2004a), providing further morphological evidence for weaker wind features in SMC stars when compared to their Galactic counterparts. While the intensity of the P Cygni emission is reduced in B-type supergiants in the SMC (Fig. 1), the terminal velocities $\left(v_{\infty}\right)$ are not significantly slower (Evans et al. 2004b), consistent with the weak metallicity-dependence predicted by theory, $v_{\infty} \propto Z^{0.13}$ (Leitherer et al. 1992); in the O-type domain this relation manifests itself more clearly, e.g., Walborn et al. (1995). 
The ratio of terminal velocity to the stellar escape velocity $\left(v_{\infty} / v_{\text {esc }}\right)$ for the SMC stars was used to show that the "bi-stability jump" in the behaviour of stellar winds at $21,000 \mathrm{~K}$ (Lamers et al. 1995) is a more gentle transition than previously thought (Evans et al. 2004b). Indeed, a more quantitative treatment of Galactic B-type supergiants found a comparable trend (Crowther et al. 2006), directly relating this to the distinctive change seen in the UV morphology between B0.5 and B0.7 subtypes (Walborn \& Nichols-Bohlin 1987). Note that the STIS data have much broader applications as a metal-poor spectral library, useful in the context of disentangling the integrated-light observations of distant super-star-clusters (e.g., Vázquez et al. 2004). They have also been incorporated in the population synthesis code STARBURST99 (Leitherer et al. 2001).

Far-UV, high-resolution spectroscopy from FUSE provided access to a wealth of additional diagnostic lines (Walborn et al. 2002). In many cases the FUSE spectra have enabled the first precise measurements of $v_{\infty}$ for stars previously with only HST-FOS or IUE data (e.g., Crowther et al. 2002; Evans et al. 2004c), as well as detailed studies of the wind structure and ionization (Massa et al. 2003). It also transpires that the P V and S IV lines in the far-UV are sensitive to the stratification of the winds, providing diagnostics of their "clumpiness" (Crowther et al. 2002; Evans et al. 2004c; Fullerton et al. 2006).

\section{Spectral analysis with improved model atmospheres}

The continued development of model atmosphere codes has also been a crucial ingredient to studies of massive stars over the past decade. The most commonly used non-LTE, line-blanketed codes that take into account spherical geometry and the effects of the stellar wind are CMFGEN (Hillier \& Miller 1998; Hillier et al. 2003) and FASTWIND (Santolaya-Rey et al. 1997; Puls et al. 2005); both codes also include parameterizations for the effects of clumping in the wind. Compared to previous results (e.g., Vacca et al. 1996), these developments led to a downward revision of the temperature scale for a given spectral type (e.g., Martins et al. 2002; Crowther et al. 2002; Repolust et al. 2004).

In cases where the winds are less significant, plane-parallel model atmospheres from TLUSTY (Hubeny \& Lanz 1995) are also used widely in the analysis of early-type stars. Even in the case of B-type supergiants, which have relatively extended atmospheres, if one excludes the features most influenced by the stellar wind (e.g., $\mathrm{H} \alpha$ and $\mathrm{H} \beta$ ), good agreement was found in the atmospheric parameters and chemical abundances obtained from TLUSTY models compared with results obtained with FASTWIND (Dufton et al. 2005).

The combination of new UV data, high-resolution optical spectroscopy and improved model atmospheres led to a number of multi-wavelength analyses of individual O-type stars in the Clouds: Crowther et al. (2002); Hillier et al. (2003); Bouret et al. (2003); Evans et al. (2004c); Heap et al. (2006). The wind properties and chemical abundances of B-type supergiants in the SMC were also investigated (Trundle et al. 2004; Trundle \& Lennon 2005).

While these studies began to explore some of the questions posed regarding stellar evolution in the Clouds, they lacked sufficiently large samples, in terms of the sampling of spectral types and luminosities. The FASTWIND analyses of 40 O-type stars from Massey et al. $(2004,2005)$ went some way to address the broader questions in the SMC compared to Galactic samples (see subsequent sections), but there remained a strong desire for a large, homogeneous sample which also included observations of early B-type stars to investigate the effects of rotation and rotationally-induced mixing.

\section{The $V L T$-FLAMES survey of massive stars}

The delivery of the FLAMES instrument to the VLT in 2002 was the catalyst for an ESO Large Programme (P.I. Smartt) to investigate the role of metallicity in the 
Table 1. Present-day composition of the LMC and SMC, as traced by early B-type stars observed in the FLAMES survey. Abundances are given on the scale $12+\log [\mathrm{X} / \mathrm{H}]$, with the relative fraction compared to the Solar results (Asplund et al. 2005) given in brackets.

\begin{tabular}{lccccc}
\hline Element & Solar & \multicolumn{2}{c}{ LMC } & \multicolumn{2}{c}{ SMC } \\
\hline $\mathrm{C}$ & 8.39 & 7.73 & {$[0.22]$} & 7.37 & {$[0.10]$} \\
\hline $\mathrm{N}$ & 7.78 & 6.88 & {$[0.13]$} & 6.50 & {$[0.05]$} \\
\hline $\mathrm{O}$ & 8.66 & 8.35 & {$[0.49]$} & 7.98 & {$[0.21]$} \\
\hline $\mathrm{Mg}$ & 7.53 & 7.06 & {$[0.34]$} & 6.72 & {$[0.15]$} \\
\hline $\mathrm{Si}$ & 7.51 & 7.19 & {$[0.48]$} & 6.79 & {$[0.19]$} \\
\hline $\mathrm{Fe}$ & 7.45 & 7.23 & {$[0.51]$} & 6.93 & {$[0.27]$} \\
\hline
\end{tabular}

evolution of massive stars. Seven fields centred on stellar clusters were observed: NGC 3293, NGC 4755, and NGC 6611 in the Galaxy; NGC 2004 and N 11 in the LMC; NGC 330 and NGC 346 in the SMC. In total, high-resolution spectroscopy was obtained for $\sim 700$ O- and early B-type stars (Evans et al. 2005, 2006). All of the observed OB-type stars have now been analysed to yield physical parameters, chemical compositions and rotational velocities, as summarised by Evans et al. (2008). Some of the key results from the survey are described in the rest of this review, combined with new results from other studies.

\subsection{Present-day composition of the $L M C \& S M C$}

Determinations of chemical abundances in rapidly-rotating stars are complicated by their broadened lines - in part the reason why previous observational effort has focused mostly on narrow-lined (i.e. slowly-rotating) stars. To inform analysis of the whole FLAMES sample, the narrow-lined B-type stars $\left(v \sin i<100 \mathrm{~km} \mathrm{~s}^{-1}\right)$ were analysed first (Hunter et al. 2007; Trundle et al. 2007), yielding stellar abundances for 87 stars in the Clouds. The present-day composition of the Clouds, as traced by these B-type stars is listed in Table 1 (Mokiem et al. 2007b). Note that due to uncertainties in the absolute abundances, the fractions quoted for iron are relative to the Galactic results from the FLAMES survey.

Given the strong evolutionary effects on nitrogen enrichment, it is difficult to obtain the pristine value. However, the lowest abundances from the FLAMES results are in good agreement with estimates from H II regions, leading to their adoption here (see discussion by Hunter et al. 2007). The oxygen abundances are in excellent agreement with results from $\mathrm{H}$ II regions, e.g., $12+\log [\mathrm{O} / \mathrm{H}]=8.35$ and 8.03 for the LMC and SMC, respectively, from Russell \& Dopita (1992). It has been known for some time that the initial abundances of carbon and nitrogen are relatively more under-abundant than the heavier elements in the Clouds; the FLAMES results reinforce the varying fractions from element to element. Indeed, simply scaling solar abundances for quantitative work in the Clouds does not best reproduce the observed patterns.

\section{Metallicity-dependent stellar winds}

Analysis of O-type spectra can be a complex, time-consuming process. In addition to the usual parameters used to characterise a star (temperature, luminosity, gravity, chemical abundances), we also need to describe the velocity structure and mass-loss rate of the wind. The analysis of the FLAMES data used a semi-automated approach, 


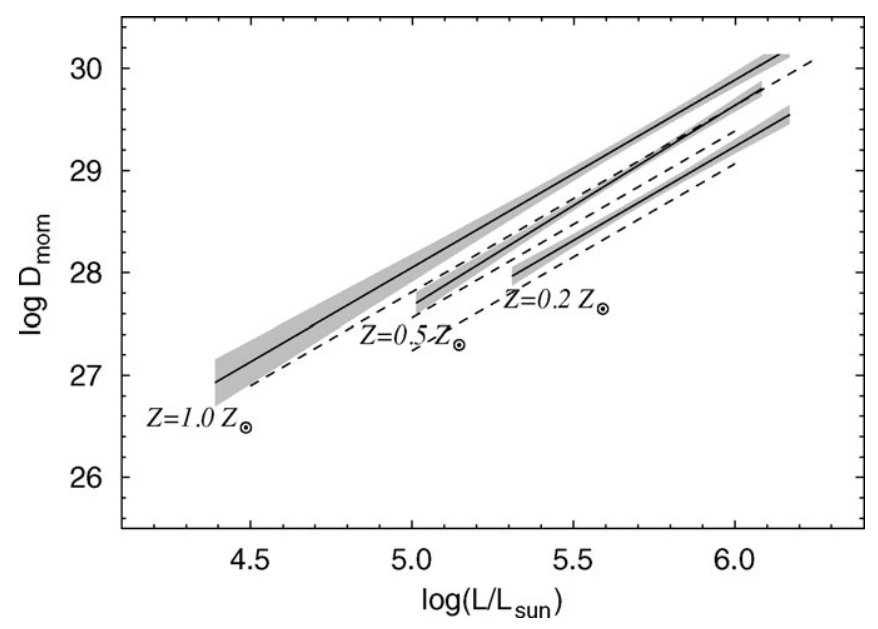

Figure 2. Comparison of the observed wind-momentum-luminosity relations (solid lines) with theoretical predictions (dashed lines) for O-type stars from Mokiem et al. (2007b). The upper, middle and lower relations correspond to Galactic, LMC and SMC results, respectively.

employing genetic algorithms (GA) to fit the observations with synthetic spectra from FASTWIND model atmospheres. This method was tested using a sample of Galactic stars, finding good agreement with previous results (Mokiem et al. 2005). The O-type spectra from the FLAMES survey were then analysed using the GA approach (Mokiem et al. 2006, 2007a).

The effect of metallicity on wind intensities in O-type stars was investigated by Massey et al. (2005). From comparisons of the wind-momenta $\left(\mathrm{D}_{\mathrm{mom}}\right.$, a function of the massloss rate, terminal velocity and stellar radius) for a sample of 22 stars in the Clouds, Massey et al. found evidence for an offset with $Z$. This result is seen more clearly in the FLAMES results, as shown in Fig. 2, providing compelling evidence for reduced intensities at decreased metallicities. Fig. 2 also shows the theoretical predictions using the prescription from Vink et al. (2001). The relative separations are in good agreement, with the FLAMES results finding a scaling of $Z^{0.72-0.83}$ (with the exponent depending on assumptions regarding clumping in the winds), as compared to $Z^{0.69 \pm 0.10}$ from theory (Mokiem et al. 2007b).

There is also quantitative evidence for weaker winds in early B-type supergiants in the SMC compared to their Galactic counterparts - somewhat reassuring given that they are the direct descendants of massive O-type stars! Fig. 3 shows the SMC results from Trundle et al. (2004) and Trundle \& Lennon (2005), compared to Galactic results from Crowther et al. (2006).

These observational tests are important for a number of areas-including considerations of the feedback from massive stars to the local interstellar medium in the context of star formation (see review by Oliveira, these proceedings). The reduced mass-loss rates at lower $Z$ mean that less angular momentum is lost, i.e. an evolved star in the SMC would be expected to retain a larger fraction of its initial rotational velocity compared to a similar star in the Galaxy. Indeed, the rotational velocity distribution for the unevolved (i.e. luminosity class IV or V) SMC stars, appears to have preferentially faster velocities when compared to Galactic results (albeit limited in terms of its statistical significance; Mokiem et al. 2006). This could offer a potential channel for long duration, gamma-ray bursts at low metallicity (e.g., Yoon et al. 2006). 

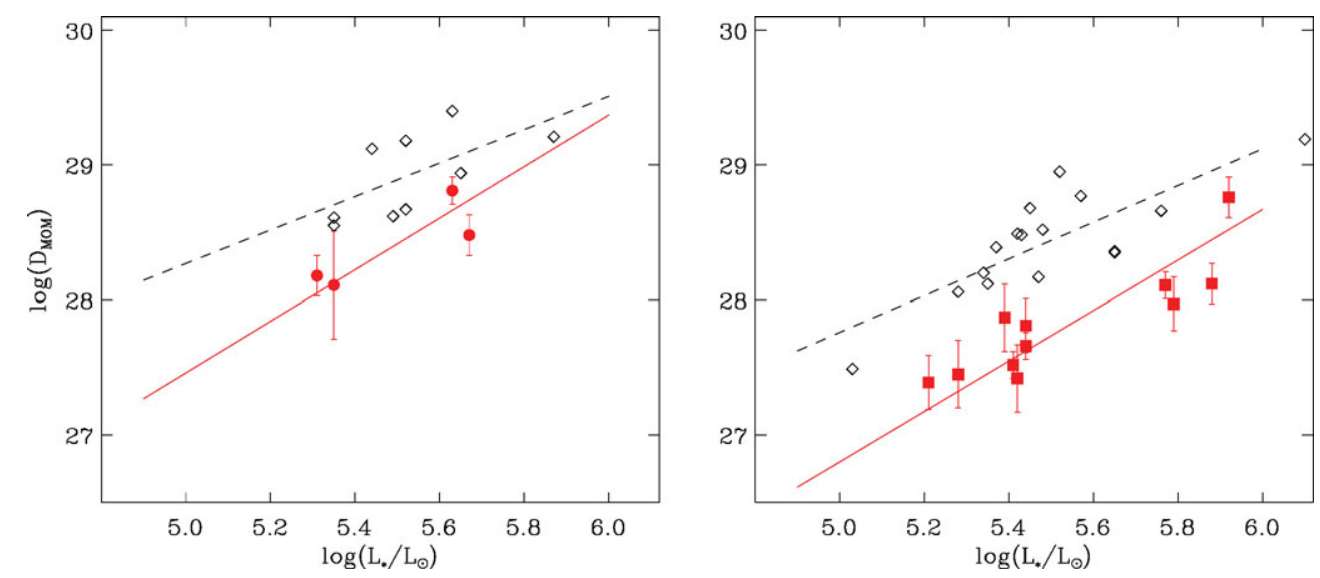

Figure 3. Observed wind-momentum-luminosity relations for early B-type supergiants (left: $\mathrm{T}_{\text {eff }}>23.5 \mathrm{kK}$; right: $\mathrm{T}_{\text {eff }}<23.5 \mathrm{kK}$ ) in the SMC (solid line, filled circles/squares) from Trundle et al. (2004) and Trundle \& Lennon (2005), compared to Galactic results (dashed line, open diamonds) from Crowther et al. (2006). Figure from Trundle et al. (2006).

Reduced mass-loss also impacts on the end products of massive O-type stars. As summarised by Crowther (2007), in the "Conti Scenario" stars in the Milky Way can expect to pass through the following phases, depending on their initial masses $\left(\mathrm{M}_{\mathrm{i}}\right)$ :

- $\mathrm{M}_{\mathrm{i}}>75 \mathrm{M}_{\odot}: \mathrm{O} \rightarrow \mathrm{WN}(\mathrm{H}-$ rich $) \rightarrow \mathrm{LBV} \rightarrow \mathrm{WN}(\mathrm{H}$-poor $) \rightarrow \mathrm{WC} \rightarrow$ SNIc;

- $\mathrm{M}_{\mathrm{i}}=40-75 \mathrm{M}_{\odot}: \mathrm{O} \rightarrow \mathrm{LBV} \rightarrow \mathrm{WN}(\mathrm{H}-$ poor $) \rightarrow \mathrm{WC} \rightarrow \mathrm{SNIc}$;

- $\mathrm{M}_{\mathrm{i}}=25-40 \mathrm{M}_{\odot}: \mathrm{O} \rightarrow \mathrm{LBV} / \mathrm{RSG} \rightarrow \mathrm{WN}(\mathrm{H}-$ poor $) \rightarrow \mathrm{SNIb}$.

With reduced mass-loss, the threshold to reach the WC phase in the SMC will move upwards from $40 \mathrm{M}_{\odot}$. This is reflected in the small relative number of WC to WN stars at low metallicity, as compared to the ratio seen in, e.g., the solar neighbourhood and M 31 (see Fig. 8 from Crowther 2007).

Moreover, a clearer picture is emerging of the progenitors of core-collapse supernovae, with increasing evidence that type II-P supernovae are descendant from red supergiants (see discussion by Smartt et al. 2008, and references therein). As such, one would expect that the ratio of type II to type Ib/c supernovae also varies with $Z$, with initial evidence of this recently reported by Prieto et al. (2008).

\section{The stellar temperature scale as a function of metallicity}

\subsection{O- and early B-type stars}

Stellar temperatures have long been known to depend on luminosity class - supergiants, with their lower gravities and extended atmospheres, are found to be cooler than dwarfs of the same spectral type. The inclusion of line-blanketing effects in the calculation of model atmospheres led to an overall downward revision of the stellar temperature scale (see Section 3). However, this effect is less dramatic at lower metallicities because of the diminished cumulative opacity from the metal lines - there is less "back warming" by trapped radiation, and so a hotter model is required to reproduce the observed spectral line-ratios (ionization balance). This effect is clearly seen in the temperatures obtained for O-type stars in the SMC, which are hotter than those found for Galactic stars with the same spectral type (Massey et al. 2005; Mokiem et al. 2006; Fig. 2). The temperatures for stars in the LMC are seen to fall neatly between the SMC and Galactic results (Mokiem 


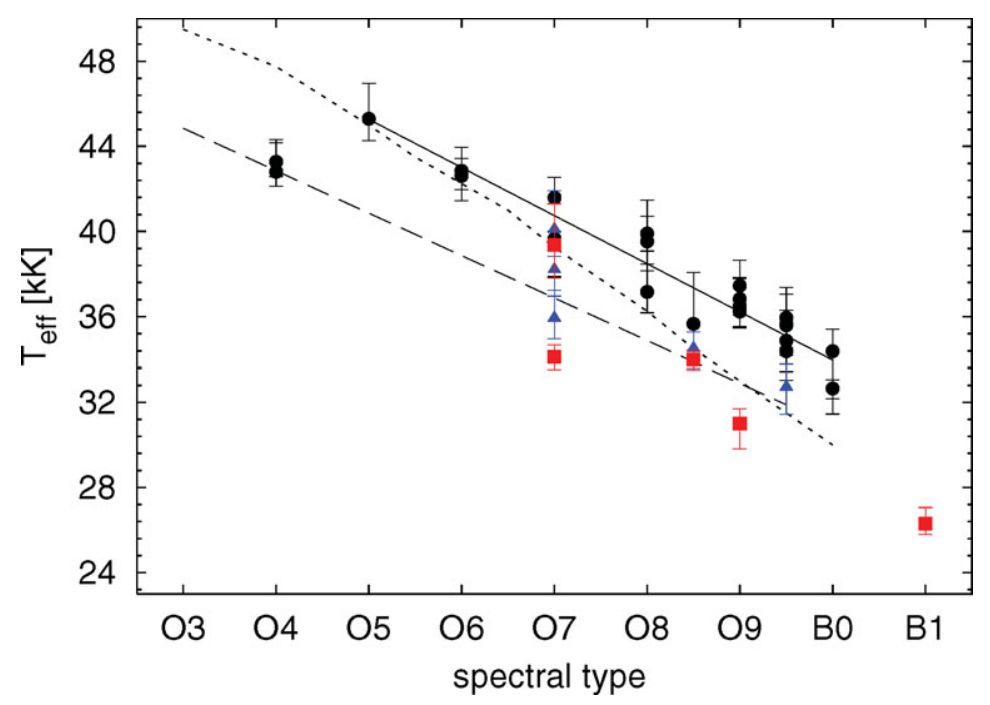

Figure 4. Effective temperatures for the O-type SMC stars analysed by Mokiem et al. (2006). The solid line is the fit to the SMC dwarfs, compared to the calibration for Galactic dwarfs from Martins et al. (2005). The dotted line is the SMC scale from Massey et al. (2005) for luminosity class $\mathrm{V}$ and III stars.

et al. 2007a). A similar $Z$-dependence has also been seen for the first time in the early B-type stars observed with FLAMES (Trundle et al. 2007).

\subsection{Late-type supergiants}

The classification of $\mathrm{O}$ - and early B-type stars is based, primarily, on the relative lineratios of different ionization stages of the same element (helium and also silicon). The $Z$-dependence of the temperature scale is a consequence of the abundance effects in the model atmosphere calculations.

For evolved, luminous supergiants there is a more direct effect of the metal abundances on the stellar temperature scale. For instance, the primary classification criterion in the A-type domain is the ratio of the $\mathrm{Ca} \mathrm{K}$ line to the blend of the $\mathrm{Ca} \mathrm{H}$ and the $\mathrm{H} \epsilon$ line. Thus, a cooler temperature is needed to reproduce the criterion for a given spectral type at SMC metallicity than in a Galactic star. This effect is the origin of the cooler temperatures reported by Venn (1999), which led her to reclassify those stars. However, the classifications should be employed on purely morphological grounds, i.e. $\mathrm{T}_{\text {eff }}$ can be $f(Z)$ at a given spectral type, but the spectral type should be independent of environment. Evans \& Howarth (2003) investigated the scale of this effect, finding lower temperatures in the SMC by up to $10 \%$ (Fig. 5). This effect, although less significant, is also seen in F- and G-type supergiants.

Temperatures determined recently for M-type supergiants in the Galaxy and the Clouds also find a $Z$-dependence, again with the SMC stars cooler at a given type (Levesque et al. 2005, 2006, 2007). The primary classification criterion in this domain is the intensity of the $\mathrm{TiO}$ bands, so cooler temperatures are also required to yield the intensity necessary for a given spectral type - an effect first noted by Humphreys (1979), while attempting to explain the later types seen in M-type supergiants in the Milky Way and LMC compared to the SMC. A similar trend in temperatures is also seen between the LMC and SMC results for K-type supergiants although, somewhat intriguingly, the Galactic stars appear slightly cooler at the early K types (Fig. 6, Levesque et al. 2007). 


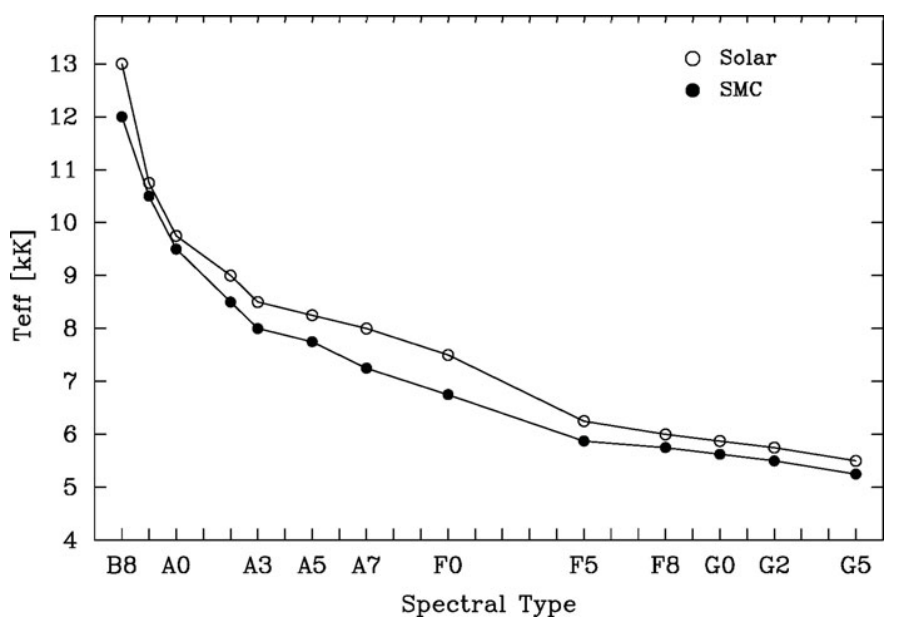

Figure 5. Temperature calibrations for late-type supergiants (Evans \& Howarth 2003).

In summary, effective temperatures (for a given spectral type) are a function of metallicity over the whole of the upper Hertzsprung-Russell diagram - from the most massive O-type stars, through to the coolest M-type supergiants. The typical variations between solar and SMC metallicity are relatively small ( $\sim 5-10 \%)$, but should be considered when adopting temperature estimates on the basis of a known spectral type.

\section{Evidence for faster rotation at low $Z$}

The first large study of the $Z$-dependence of stellar rotational velocities was the work by Keller (2004), who observed $\sim 100$ B-type stars in the LMC and compared their rotation rates with published Galactic results. Keller found that the cluster members had mean rotational velocities that were larger than field stars (at the corresponding metallicity), and that the LMC cluster members were rotating more quickly than those in Galactic clusters (at just under a $2 \sigma$ significance). Since then, a number of surveys have used FLAMES to investigate these effects further in early B-type stars (in which the effects of mass-loss are relatively small, thereby removing a further complication to the evolution of $v \sin i$ ).

From FLAMES observations of B- and Be-type stars in fields centred on NGC 330 and NGC 2004, Martayan et al. (2007) found evidence for faster rotational velocities at the lower metallicity of the SMC. Similar conclusions were found from the analysis of Hunter et al. (2008a; Fig. 5), in which the SMC stars are found to rotate more quickly than those in the Galaxy, with a significance at the $3 \sigma$ level. Given the overlapping target fields, an independent check on the methods was possible between the results of Martayan et al. and Hunter et al., finding good agreement in the velocity distributions of normal B-type stars in the NGC 330 field.

To complicate this picture slightly, new results from additional FLAMES observations (Royer et al., this meeting ) find velocity distributions in the LMC and SMC that are consistent with being drawn from the same parent population - this raises the question of whether local metallicity variations could account for the differences? Given the large global offset between the LMC and SMC, this seems relatively unlikely, but it appears that something is still eluding us at the current time!

\section{Reconciling the 'Hunter diagram' with evolutionary predictions}

To investigate the impact of rotation on surface nitrogen abundances in the FLAMES sample, new evolutionary models were calculated using the chemical compositions from 


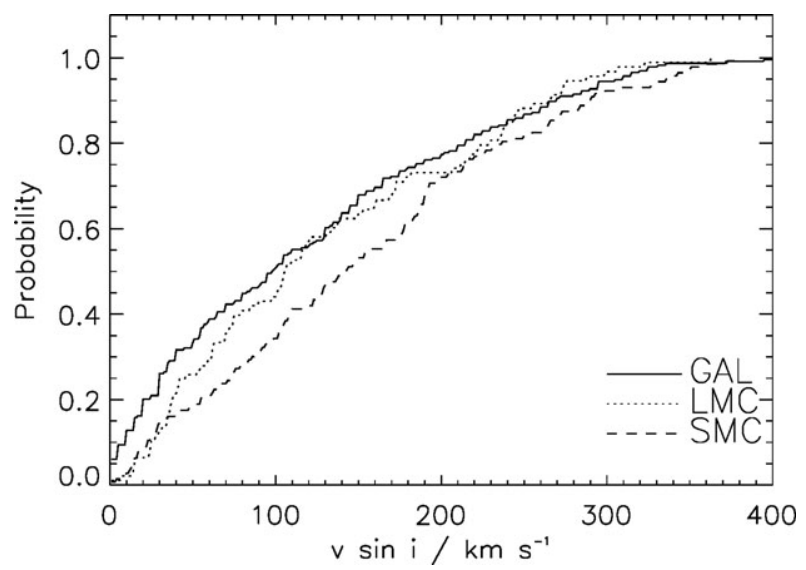

Figure 6. Cumulative distribution functions for the rotational velocities of Galactic field stars, compared with LMC and SMC results from FLAMES (Hunter et al., 2008a).

Table 1. Fig. 6 shows the nitrogen abundances, as a function of $v \sin i$ for the B-type stars in the LMC from the FLAMES survey (Hunter et al. 2008b). Typical uncertainties in the abundances are $\sim 0.2-0.3 \mathrm{dex}$, so the scatter of the results indicates genuine differences in the N-enrichment in both the core-hydrogen-burning stars (dwarfs and giants, left-hand panel) and the supergiants (right-hand panel).

Two groups ("Groups 1 and 2") appear inconsistent with the predicted abundances. The dark grey (blue) points in Group 1 comprise rapidly-rotating stars that appear to have undergone little chemical mixing, and yet they have surface gravities that indicate they are near the end of core hydrogen burning. These results are at odds with the evolutionary models that predict nitrogen abundances some $\sim 0.5$ dex greater for the more massive stars (in which mixing is expected to be most efficient). Note that there was no evidence for binarity in the spectra of many of these stars.

The 14 (apparently single) core-hydrogen-burning stars in Group 2 are equally puzzling as they are rotating very slowly $\left(v \sin i<50 \mathrm{~km} \mathrm{~s}^{-1}\right)$, but show significant N-enrichment. For a random orientation, we could expect about two of these to be rapidly-rotating stars viewed pole-on. This seems an unlikely explanation for all 14, and Hunter et al. concluded that the majority are intrinsically slow rotators. Recent studies of Galactic $\beta$-Cepheid stars have found a correlation between nitrogen enrichment and magnetic fields (Morel et al. 2006); perhaps the enrichments in Group 2 are related to magnetic fields.

The results for the supergiants can be considered as two groups - Group 3, with relatively normal levels of N-enrichment, and Group 4, with much larger abundances. Simplistically these could be pre-RSG (Group 3) and post-RSG (Group 4) stars. However, while the abundances of the Group 4 stars are consistent with the predicted level, the models cannot reproduce their effective temperature in the Hertzsprung-Russell diagram. Some of these show evidence of binarity, so mass transfer may also be important.

So, rotationally-induced mixing appears to play a key role in the enrichment of surface nitrogen in massive stars, but it appears that there are also other processes at work, particularly at low rotational velocities - presenting new challenges to the evolutionary models.

\section{Massive binaries}

The effects of binarity/multiplicity on the formation and subsequent evolution of highmass stars are a vibrant area of research. One of the key ingredients missing from 


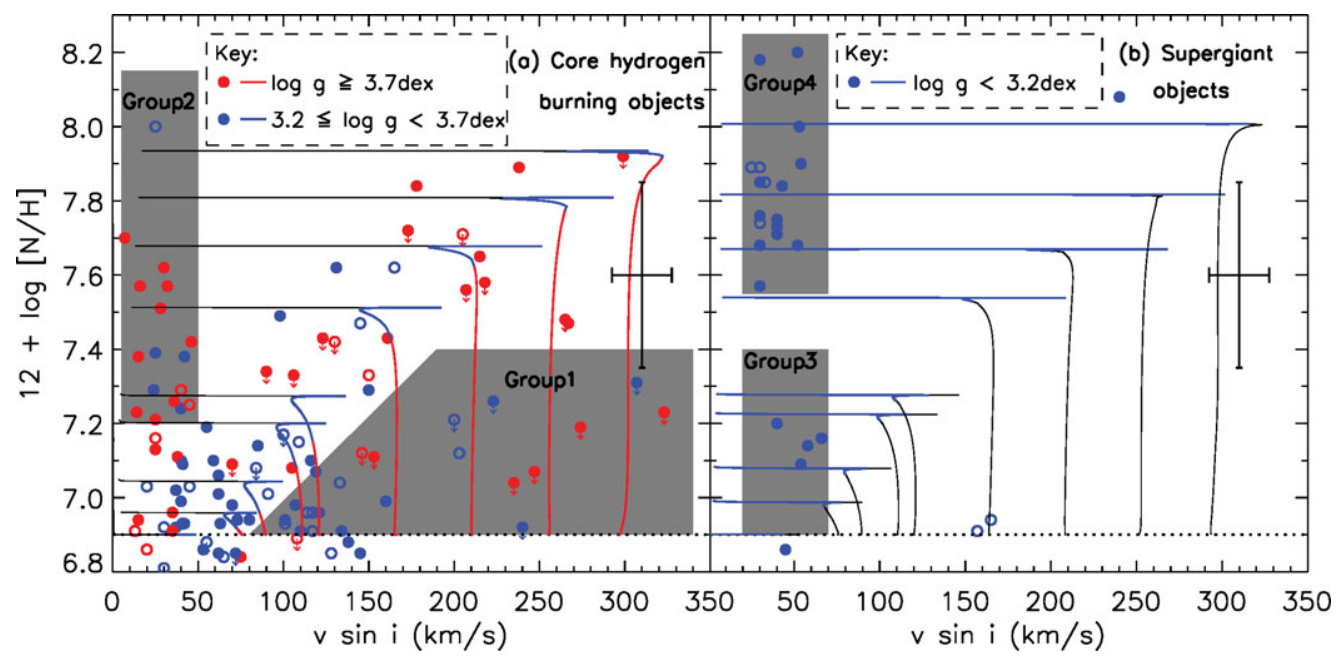

Figure 7. Nitrogen abundances $(12+\log [\mathrm{N} / \mathrm{H}])$ compared to projected rotational velocities for core-hydrogen-burning (left-hand panel) and supergiant (right-hand panel) B-type stars in the LMC (Hunter et al. 2008b). The solid lines are new evolutionary tracks, open circles are radial velocity variables, downward arrows are upper limits, and the dotted horizontal line is the LMC baseline nitrogen abundance.

current theories of both star formation and cluster evolution is a robust binary fraction of high-mass stars (and the distribution of the relative mass-ratios in these systems). Observational effort in this area has been somewhat piecemeal to date (e.g., Sana et al. 2008), with Zinnecker \& Yorke (2007) highlighting the need for multi-epoch radial velocity surveys of stellar clusters to provide better constraints to theoretical models of star formation.

One of the serendipitous aspects of the FLAMES survey was the large number of spectroscopic binaries discovered. The time sampling of the service-mode observations did a reasonable (but not thorough) job of binary detection in three of the fields in the Clouds, with lower limits to the binary fraction of 25-35\% (Evans et al. 2006). As illustrated by new multi-epoch observations in 30 Doradus with GMOS (Bosch et al., these proceedings), the true binary fraction in young clusters could be much larger.

\section{Closing remarks}

There has been huge observational and theoretical progress in our understanding of massive star evolution in the Clouds in the past decade. The intensity of stellar winds, the effective temperature scale, and the rotational velocities of OB-type stars are all found to be dependent on the metal content of their local environment.

Multi-object spectrographs (e.g., FLAMES, 2dF and GMOS) have truly opened-up our knowledge of the stellar content of the Clouds over the past decade. We are beginning to discover many new examples of the peculiar members of the 'OB Zoo' (e.g., Vz, f?p, nfp, B[e], etc.) and with future surveys we will be able to explore the evolutionary connection of these rare sub-types to the morphologically normal population - are they critical, short-lived phases that every massive star experiences?

Moreover, key questions remain regarding the binary fraction of massive stars, binary evolution, and the efficiency of rotationally-induced mixing in O-type stars. The VLTFLAMES Tarantula Survey is an ESO Large Programme (P.I. Evans) approved in July 
2008 to address these issues, via multi-epoch observations of $\sim 1,000$ stars in 30 Doradus in the LMC.

Lastly, it is worth noting that IUE left a large spectral archive of Galactic OB-type stars, which has continued to yield new results. While FUSE has observed a large number of early-type stars (giving us a valuable window on the wind parameters revealed in the far-UV), it is remarkable that high-resolution spectroscopy from HST exists for only $\sim 40$ stars in the Clouds. The 1200-1900 $\AA$ region is uniquely important in the study of massive stars and, given the absence of future UV missions at the advanced stages, new $H S T$ observations after the servicing mission would provide immense legacy value.

\section{Acknowledgements}

Grateful thanks to Nolan Walborn for careful reading of this manuscript.

\section{References}

Asplund, M., Grevesse, N. \& Sauval, A. J. 2005, in: T. G. Barnes III \& F. Bash (eds.), Cosmic Abundances as Records of Stellar Evolution and Nucleosynthesis, ASP Conf. Series, Vol. 336, p. 25

Bouret, J.-C., Lanz, T., Hillier, D. J., Heap, S. R., Hubeny, I., Lennon, D. J., Smith, L. J., \& Evans, C. J. 2003, ApJ, 595, 1182

Crowther, P. A., Hillier, D. J., Evans, C. J., Fullerton, A. W., De Marco, O., \& Willis, A. J. 2002, ApJ, 579, 774

Crowther, P. A., Lennon, D. J., \& Walborn, N.R. 2006, A\& A, 446, 279

Crowther, P. A. 2007, ARAA, 45, 177

Dufton, P. L., Ryans, R. S. I., Trundle, C., Lennon, D. J., Hubeny, I., Lanz, T., \& Allende Prieto, C. 2005, A\&BA, 434, 1125

Evans, C. J. \& Howarth, I. D. 2003, MNRAS, 345, 1223

Evans, C. J., Lennon, D. J., Walborn, N. R., Trundle, C., \& Rix, S. A. 2004a, PASP, 116, 909

Evans, C. J., Lennon, D. J., Trundle, C., Heap, S. R., \& Lindler, D. J. 2004b, ApJ, 607, 451

Evans, C. J., Crowther, P. A., Fullerton, A. W., \& Hillier, D. J. 2004c, ApJ, 610, 1021

Evans, C. J., Smartt, S. J., Lee, J.-K, et al. 2005, A\&\&A, 437, 467

Evans, C. J., Lennon, D. J., Smartt, S. J., \& Trundle, C. 2006, A\& A, 456, 623

Evans, C. J, Hunter, I., Smartt, S., et al. 2008, Msngr, 131, 25

Fullerton, A. W., Massa, D. L., \& Prinja, R. K. 2006, ApJ, 637, 1025

Grebel, E. K., Richtler, T., \& de Boer, K. S. 1992, A\&A, 254, L5

Heap, S. R., Lanz, T., \& Hubeny, I. 2006, ApJ, 638, 409

Heger, A. \& Langer, N. 2000, ApJ, 544, 1016

Hillier, D. J. \& Miller, D. L. 1998, ApJ, 496, 407

Hillier, D. J., Lanz, T., Heap, S. R., Hubeny, I., Smith, L. J., Evans, C. J., Lennon, D. J., \& Bouret, J. C. 2003, ApJ, 588, 1039

Hubeny, I. \& Lanz, T. 1995, ApJ, 439, 875

Humphreys, R. M. 1979, A\& A, 231, 384

Hunter, I., Dufton, P. L., Smartt, S. J., et al. 2007, A\&A, 466, 277

Hunter, I., Lennon, D. J., Dufton, P. L., Trundle, C., Simn-Daz, S., Smartt, S. J., Ryans, R. S. I., \& Evans, C. J. 2008a, A\&A, 479, 541

Hunter, I., Brott, I., Lennon, D. J., et al. 2008b, $A \mathscr{E} A$, 676, L29

Keller, S. C. 2004, PASA, 21, 310

Kudritzki, R.-P., Pauldrach, A., \& Puls, J. 1987, A\&A, 173, 293

Lamers, H. J. G. L. M., Snow, T. P., \& Lindholm, D. M. 1995, ApJ, 455, 269

Leitherer, C., Robert, C., \& Drissen, L. 1992, ApJ, 401, 596

Leitherer, C., Leão, J. R. S., Heckman, T. M., Lennon, D. J., Pettini, M., \& Robert, C. 2001, ApJ, 550, 724

Levesque, E. M., Massey, P., Olsen, K. A. G., Plez, B., Josselin, E., Maeder, A., \& Meynet, G. 2005, ApJ, 628, 973 
Levesque, E. M., Massey, P., Olsen, K. A. G., Plez, B., Meynet, G., \& Maeder, A. 2006, ApJ, 645,1102

Levesque, E. M., Massey, P., Olsen, K. A. G., \& Plez, B. 2007, ApJ, 667, 202

Maeder, A., Grebel, E. K., \& Mermilliod, J.-C. 1999, A\& A, 346, 459

Maeder, A. \& Meynet, G. 2001, A\&A A, 373, 555

Martayan, C., Frémat, Y., Hubert, A.-M., Floquet, M., Zorec, J., \& Neiner, C. 2007, A\&\&A, 462, 683

Martins, F., Schaerer, D., \& Hillier, D.J. 2002, A\&A A, 382, 999

Martins, F., Schaerer, D., \& Hillier, D. J. 2005, A\&A, 436, 1049

Massa, D., Fullerton, A. W., Sonneborn, G., \& Hutchings, J. B. 2003, ApJ, 586, 996

Massey, P., Bresolin, F., Kudritzki, R. P., Puls, J., \& Pauldrach, A. W. A. 2004, ApJ, 608, 1001

Massey, P., Puls, J., Pauldrach, A. W. A., Bresolin, F., Kudritzki, R. P., \& Simon, T. 2005, ApJ, 627, 477

Meynet, G. \& Maeder, A. 2000, A\& A, 361, 101

Mokiem, M. R., de Koter, A., Puls, J., Herrero, A., Najarro, F., \& Villamariz, M. R. 2005, A\&্A, 441,711

Mokiem, M. R., de Koter, A., Evans, C. J., et al. 2006, A\&SA, 456, 1131

Mokiem, M. R., de Koter, A., Evans, C. J., et al. 2007a, A\&A, 465, 1003

Mokiem, M. R., de Koter, A., Vink, J. S., et al. 2007b, $A \& A$, 473, 603

Morel, T., Butler, K., Aerts, C., Neiner, C., \& Briquet, M. 2006, A $\mathscr{E} A$, 457, 651

Prieto, J. L., Stanek, K. Z., \& Beacom, J. F. 2008, ApJ, 673, 999

Prinja, R. K. \& Crowther, P. A. 1998, MNRAS, 300, 828

Puls, J., Urbaneja, M. A., Venero, R., Repolust, T., Springmann, U., Jokuthy, A., \& Mokiem, M. R. 2005, $A \& A, 435,669$

Repolust, T., Puls, J., \& Herrero, A. 2004, A\&A, 415, 349

Russell, S. C. \& Doptia, M. A. 1992, ApJ, 384, 508

Sana, H., Gosset, E., Nazé, Y., Rauw, G., \& Linder, N. 2008, MNRAS, 386, 447

Santolaya-Rey, A. E., Puls, J., \& Herrero, A. 1997, A\&SA, 323, 488

Smartt, S. J., Eldridge, J. J., Crockett, R. M., \& Maund, J. R. 2008, MNRAS, submitted, arXiv:0809.0403

Trundle, C., Lennon, D. J., Puls, J., \& Dufton, P. L. 2004, A\&ऽA, 417, 217

Trundle, C. \& Lennon, D. J. 2005, A\& $A, 434,677$

Trundle, C., Lennon, D. J., Puls, J., Dufton, P. L., \& Evans, C. J. 2006, in: H. J. G. L. M. Lamers, N. Langer, T. Nugis, \& K. Annuk (eds.), Stellar Evolution at Low Metallicity: Mass Loss, Explosions, Cosmology, ASP Conf. Series, Vol. 353, p. 127

Trundle, C., Dufton, P. L., Hunter, I., Evans, C. J., Lennon, D. J., Smartt, S. J., \& Ryans, R.S.I. 2007, A\&A, 471, 625

Vacca, W. D., Garmany, C. D., \& Shull, J. M. 1996, ApJ, 460, 914

Vázquez, G. A., Leitherer, C., Heckman, T. M., Lennon, D. J., de Mello, D. F., Meurer, G. R., \& Martin, C. L. 2004, ApJ, 600, 162

Venn, K. A. 1999, ApJ, 518, 405

Vink, J. S., de Koter, A., \& Lamers, H. J. G. L. M. 2000, $A \& \& A, 362,295$

Vink, J. S., de Koter, A., \& Lamers, H. J. G. L. M. 2001, $A \& \& A, 369,574$

Walborn, N. R. \& Nichols-Bohlin, J. 1987, PASP, 99, 40

Walborn, N. R., Lennon, D. J., Haser, S. M., Kudritzki, R.-P., \& Voels, S. A. 1995, PASP, 107, 104

Walborn, N. R., Lennon, D. J., Heap, S. R., Lindler, D. J., Smith, L. J., Evans, C. J., \& Parker, J. Wm. 2000, PASP, 112, 1243

Walborn, N. R., Fullerton, A. W., Crowther, P. A., Bianchi, L., Hutchings, J. B., Pellerin, A., Sonneborn, G., \& Willis, A.J. 2002, ApJS, 141, 443

Yoon, S.-C., Langer, N., \& Norman, C. 2006, A\&A, 460, 199

Zinnecker, H. \& Yorke, H. W. 2008, ARAA, 45, 481 